\title{
An electrical circuit model of the alpha-beta merging seen in dielectric relaxation of ultraviscous liquids
}

Saglanmak, Neslihan; Nielsen, Albena; Olsen, Niels Boye; Dyre, J. C.; Niss, Kristine

Published in:

Journal of Chemical Physics

DOI:

$10.1063 / 1.3270164$

Publication date:

2010

\section{Document Version}

Publisher's PDF, also known as Version of record

Citation for published version (APA):

Saglanmak, N., Nielsen, A., Olsen, N. B., Dyre, J. C., \& Niss, K. (2010). An electrical circuit model of the alphabeta merging seen in dielectric relaxation of ultraviscous liquids. Journal of Chemical Physics, 132(2), 02450301 til 024503-12. https://doi.org/10.1063/1.3270164

\section{General rights}

Copyright and moral rights for the publications made accessible in the public portal are retained by the authors and/or other copyright owners and it is a condition of accessing publications that users recognise and abide by the legal requirements associated with these rights.

- Users may download and print one copy of any publication from the public portal for the purpose of private study or research.

- You may not further distribute the material or use it for any profit-making activity or commercial gain.

- You may freely distribute the URL identifying the publication in the public portal.

Take down policy

If you believe that this document breaches copyright please contact rucforsk@kb.dk providing details, and we will remove access to the work immediately and investigate your claim. 


\title{
An electrical circuit model of the alpha-beta merging seen in dielectric relaxation of ultraviscous liquids
}

\author{
Neslihan Sağlanmak, Albena I. Nielsen, Niels Boye Olsen, Jeppe C. Dyre, and \\ Kristine Niss ${ }^{a}$ \\ Department of Sciences, DNRF Center “Glass and Time," IMFUFA, Roskilde University, Universitetsvej 1, \\ Roskilde 4000, Denmark
}

(Received 17 July 2009; accepted 10 November 2009; published online 12 January 2010)

\begin{abstract}
We present a new model for dielectric data in the alpha-beta merging region. The model is constructed using electrical circuit analogies. It leads to an interpretation of the merging region as one where the total relaxation upon cooling separates in two relaxation processes, consistent with a view where the relaxing entities involved are the same for the two processes. We use this alpha-beta model to fit dielectric data in the merging region of two different molecular liquids. These fits are performed under the assumption that the intrinsic high-frequency behavior of the alpha relaxation is a $-1 / 2$ power law and that both the alpha and the beta process separately obey time temperature superposition. We get good quality fits in the entire frequency and temperature range studied. This supports the view that alpha relaxation high-frequency slopes that are found to be numerically smaller than 1/2 can be attributed to the influence of the beta relaxation. (C) 2010 American Institute of Physics. [doi:10.1063/1.3270164]
\end{abstract}

\section{INTRODUCTION}

Relaxation in ultraviscous liquids after a small perturbation is dominated by the primary alpha relaxation, but many frequency-temperature spectra also show one or several liquid-specific secondary beta processes which appear in the kilo- to megahertz region. Such secondary processes can also be found in rigid molecules as first demonstrated by Johari and Goldstein, ${ }^{1,2}$ and these are referred to as the JohariGoldstein beta process. The characteristic time of the primary alpha relaxation increases dramatically with decreasing temperature, while the temperature dependence of the secondary process is weaker. Therefore an alpha-beta merging or crossover temperature above the glass transition temperature $\left(T_{g}\right)$ can be identified. The fundamental phenomenological challenge in the field of ultraviscous liquids is to map out the behavior and interplay between these two relaxations. The properties which characterize them, and which are believed to have importance for understanding of the viscous slowing down, include the temperature dependences of the characteristic times, ${ }^{3-7}$ the spectral shapes, ${ }^{8,9}$ the amplitudes/ strengths of the processes, $6,10-12$ and the possible relation between the two processes. ${ }^{7,13-17,52}$ In this work we limit the discussion to the behavior found in the dielectric relaxation. Dielectric spectroscopy data play a special role in the study of the phenomenology of highly viscous liquids because they are the most abundant. It is of course important to extend conclusions to different types of probes, but first observations are often established from dielectric data.

In earlier work we focused on the shape of the alpha relaxation seen in dielectric spectroscopy. ${ }^{8,16}$ This was done with an unbiased approach where we introduced the mathematically well-defined minimum slope and a time tempera-

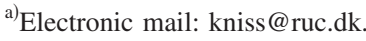

ture superposition (TTS) parameter that is directly related to the spectra instead of the more common approach of fitting the spectra to some function. The direct reference to the actual data is an advantage, because the result of a fit is often very dependent on how much of the frequency range is available and this can lead to artificial results, e.g., for the temperature dependences of the parameters. ${ }^{8,16}$ Our view, based on these earlier studies, is that the alpha relaxation broadens as temperature decreases until it reaches the slope $1 / 2$, whereafter it stays TTS in the remaining low temperature region. When the spectra appears even broader, and have slopes numerically smaller than $1 / 2$, we believe it is due to the influence of one or more underlying, low-frequency beta process(es). To substantiate this view, and in general to be able to characterize the behavior of the individual processes in the merging region, it is necessary to disentangle them from each other. However, it is not possible to separate the processes via an unbiased study of the raw data. The separation of the processes can only be done by introducing assumptions and by using a consistent fitting procedure. Our aim in this work is to do so by developing a new model for the alpha-beta merging region.

To model the two processes' interaction we need to consider two questions. The first question is how to describe the alpha and the beta relaxation, respectively. The second question is how to model the merging of the two processes' spectra. The beta process will be fitted by a Cole-Cole (CC) function, since the beta peak is symmetric. Our focus is on the new approach for handling the merging of the relaxations, but we moreover present a new description of the alpha process.

The merging of the alpha relaxation and the beta relaxation is commonly fitted either by assuming that the two spectra can be added or by using the Williams ansatz ${ }^{18-20,35}$ 
which leads to a product of the alpha and the beta relaxation functions in the time domain. The additive approach is used more often because it is the easiest from a practical point of view. The Williams approach is more complicated to use in a fitting process, and is rarely used, but most scientists in the field consider it to be the correct approach, although there is some discussion regarding the question. ${ }^{21-24}$ Looking at the original Williams paper (Refs. 18-20), it appears that he assumes the two processes to be statistically independent and that he is probably thinking of side chain motions in polymers rather than the intrinsic Johari-Goldstein beta relaxation which is found in the relaxation in rigid molecule liquids. ${ }^{1,2}$ Today there is much evidence pointing to a strong interdependence of the two processes, which suggests that a proper model should be one where the two processes are closely interlinked. ${ }^{13,15}$

In our approach, the merging region is seen as one where the total relaxation upon cooling separates in two relaxation processes, consistent with a view where the relaxing entities involved are the same for the two processes. The model has the feature that the beta process can never be seen if it is slower than the alpha process, as is also found when using the Williams ansatz. The model is expressed directly in the frequency domain and therefore well suited for fitting data from dielectric spectroscopy.

The tool we use for constructing this model is electrical circuit analogies. When using electrical circuits to model dielectric relaxation response the electrical charge and potential represent polarization and field. In this sense the circuits can be seen as a less abstract representation of the so-called network thermodynamics. ${ }^{25-27}$ The use of circuits assures that the model is physically consistent, e.g., obeys conservation of energy, positive dissipation, causality, linearity, etc. It moreover leads to an expression for the response function in the frequency domain with real and imaginary parts which obey the Kramers-Kronig relation.

The structure of the paper is as follows: in Sec. II different fitting functions for the alpha relaxation are discussed, in order to motivate the formulation of a new model for the dielectric alpha relaxation of molecular glass formers. The electrical circuit approach will be explained by building up the electrical circuit model by extension from the electrical circuit counterpart of the Debye model. The new electrical circuit model gives a function that can be used to describe the non-exponential alpha relaxation. This new expression for the primary process is interpreted and analyzed in Secs. II B and II C. In Sec. III the electrical circuit model of the $\mathrm{CC}$ function and assumptions regarding the secondary relaxation process are briefly presented.

The new alpha-beta circuit model covering the merging of the two relaxations is presented and analyzed in Sec. IV. This includes a comparison to the additive combination of the alpha and the beta relaxation, and a brief comparison to the Williams approach.

The alpha-beta circuit model is fitted to experimental dielectric data, namely, the whole temperature-frequency scans for the two liquids toluene-pyridine mixture (temperature range of 125-131 K and frequency range of $10^{-2}-10^{6.5} \mathrm{~Hz}$ ) and di-iso-butyl phthalate (temperature range of 191-221 $\mathrm{K}$ and frequency range of $10^{-2.7}$ $-10^{7.5} \mathrm{~Hz}$ ), i.e., above, at, and under the temperature at which the primary and secondary processes merge.

\section{THE MODEL FOR THE ALPHA RELAXATION}

In this section we will develop and discuss a model for the alpha relaxation which is needed for modeling the merging region. The most common fitting functions (presented in Table I) are the stretched exponential or KohlrauschWilliams-Watts (KWW) function, which is given as an explicit function in the time domain, the Cole-Davidson (CD) function, and the Havriliak-Negami function (HN) which are both functions expressed in the frequency domain. Each of these fitting functions have problems which have been discussed detailed in several papers by a number of authors. ${ }^{34,35,37-40}$ Here we shall only summarize the discussion in order to motivate the introduction of a new function.

Phenomenologically, it is well established that the imaginary part of the susceptibility of molecular viscous liquids converges toward a low-frequency power law dependence of $\omega^{1}$ and a high-frequency power law of $\omega^{-\alpha}$, where $\alpha$ takes values between zero and one depending on the liquid and the state point. This behavior is captured by both the KWW and the CD functions which both have one shape parameter, namely, the exponent governing the high-frequency slope. However, the two functions have different shapes even if the high-frequency exponent is set to the same value. The KWW is wider than the $\mathrm{CD}$ for a given value of the exponent (see, e.g., Ref. 34) and the KWW approaches the low-frequency power law much more slowly than the $\mathrm{CD}$. It is sometimes found that KWW gives good fits, though the CD seems to more often be the best choice, ${ }^{8}$ but the general picture is that the width seen in different liquids can take different values even if the high-frequency power law is the same. ${ }^{8,16}$ This means that the spectral shape needs to be described by two parameters. The HN function introduces an extra parameter, but it does not have the correct limiting low-frequency behavior. There have also been various suggestions for other ways of characterizing data. Blochowicz and co-workers ${ }^{34}$ take the strategy of describing the relaxation by a sum of Debye relaxations. This is a consistent approach and the resulting function has both real and imaginary parts, as well as the correct low-frequency behavior. However, it is a somewhat involved fitting procedure. Bergman has, in Refs. 35, 38 , and 39 , proposed a fitting function for the imaginary part of the relaxation which is constructed in order to make a peak with high and low-frequency power law dependences and varying peak width. This function, however, is a function of $\omega$ and not of $i \omega$ this means that it does not have a corresponding real part and thus we cannot know whether it corresponds to the Laplace transform of any function in the time domain.

To summarize we find that a suitable function describing the dielectric alpha relaxation should have the following three properties: (i) be expressed as a function of $i \omega$; (ii) have a low-frequency limiting power law with exponent 1 and a high-frequency power law with a variable exponent which can take values between -1 and zero; and (iii) the 
TABLE I. An overview of different fitting functions for the alpha relaxation. The parameters counted in the table are those controlling the shape; each function has in addition a time-scale and a strength parameter. The high-frequency dielectric constant is also a parameter, except in Bergman's function which only includes the dispersion.

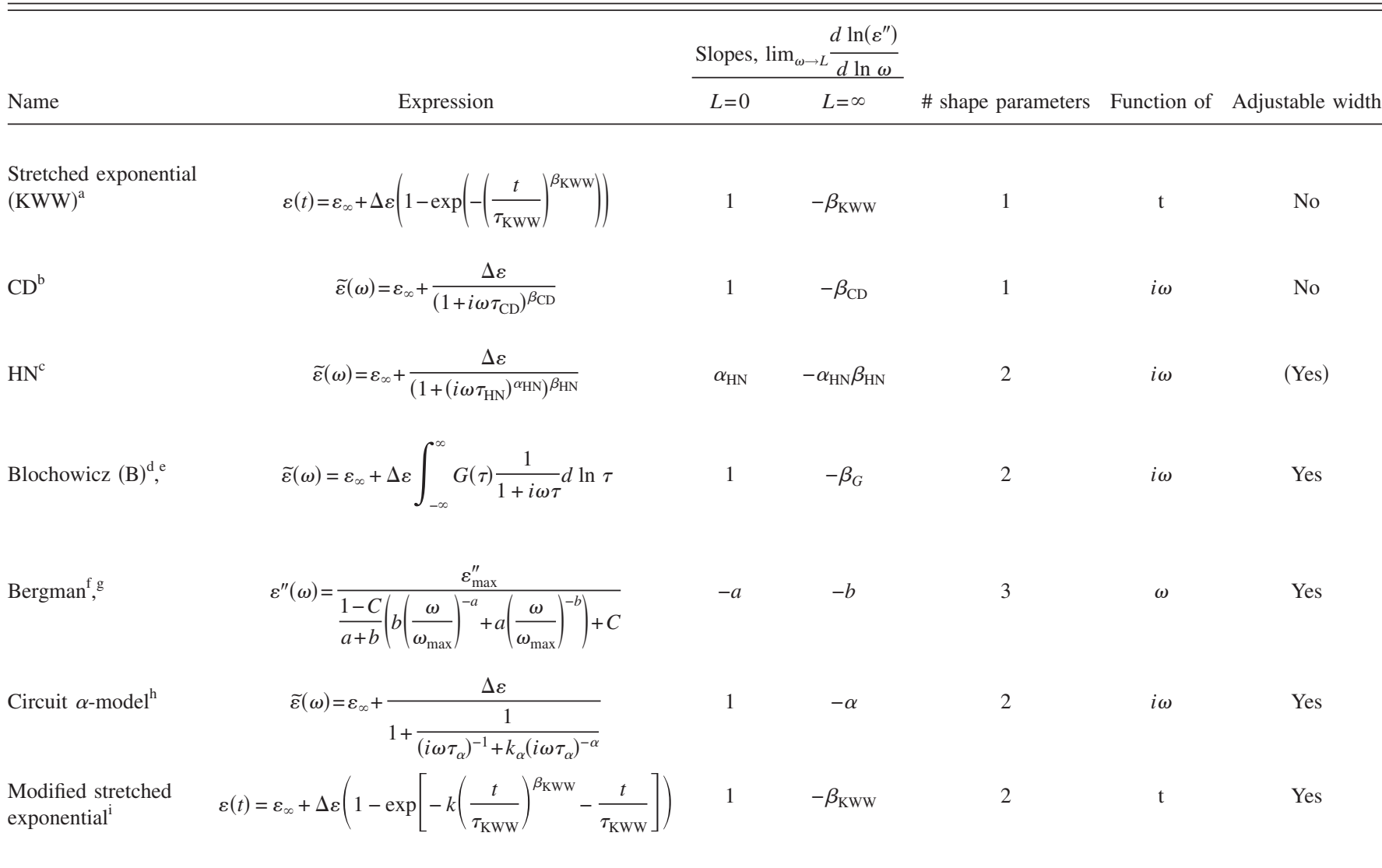

${ }^{\mathrm{a}}$ References 18,28 , and 29.

${ }^{\mathrm{b}}$ References 30 and 31 .

${ }^{\mathrm{c}}$ References 32 and 33 .

${ }^{\mathrm{d}}$ Reference 34.

${ }^{\mathrm{e}}$ In this expression the generalized gamma distribution function is $G(\tau)=N(\alpha, \beta)\left(\tau / \tau_{0}\right)^{\beta} \exp \left[-(\beta / \alpha)\left(\tau / \tau_{0}\right)^{\alpha}\right]$ and the normalization factor is $N(\alpha, \beta)$ $=(\beta / \alpha)^{\beta / \alpha} \alpha / \Gamma(\beta / \alpha)$.

${ }^{\mathrm{f}}$ References 35 and 39.

${ }^{\mathrm{g}}$ All parameters are fitted only to the dispersion. $C$ is the bluntness (width) parameter.

${ }^{\mathrm{h}} \mathrm{See}$ the text for explanation of the parameters.

${ }^{1}$ Reference 36.

width of the relaxation should be variable for fixed highfrequency exponent. In the following section we construct such a fitting function based on an electrical circuit model.

All the functions mentioned in the section above are shown in Table I. We also show a modification of the stretched exponential which fulfills requirements (ii) and (iii) but is expressed in the time domain (see Table I for the expression). We use this function to represent time domain data in Ref. 36. This time domain function is not exactly equivalent to the circuit model even if they have the same qualitative characteristics. At this point we do not know which of the two is better, but focus on the circuit model in this paper because it is better suited for frequency domain data.

\section{A. The alpha circuit model}

The simplest approach when modeling the dielectric alpha relaxation is to apply the Debye model ${ }^{41}$ in both time and frequency domains. This gives an exponential relaxation, and it will therefore not give a proper fit to the asymmetric relaxation seen in data. It is nevertheless very intuitive and we use it, as Cole and Cole did in Ref. 42, as a starting point for our electrical circuit model.

The electrical circuit leading to the Debye model is shown in Fig. 1. The complex capacitance of the circuit $\widetilde{C}$ can easily be found (remembering that the capacitance and the impedance, $\tilde{Z}(\omega)$, are related in the following way: $\widetilde{C}(\omega)=1 /(i \omega \widetilde{Z}(\omega)))$. It is given by:

$$
\widetilde{C}(\omega)=C_{1}+\frac{1}{\frac{1}{C_{2}}+i \omega R}=C_{1}+\frac{C_{2}}{1+i \omega \tau_{D}},
$$

where $\tau_{D}=R C_{2}$ the relaxation time. This expression corresponds to the well-known Debye model $^{41}$ for dielectric relaxation of liquids, 


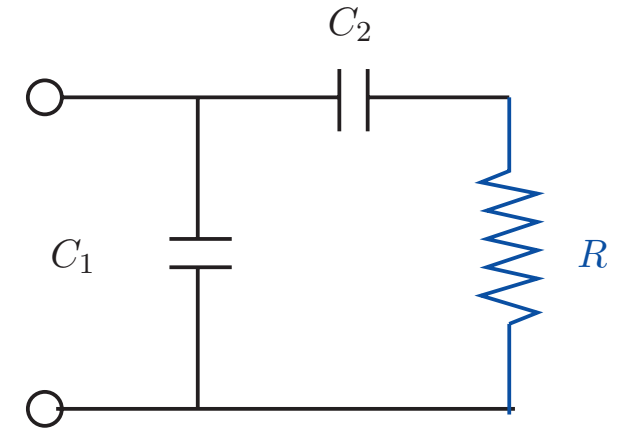

FIG. 1. Electrical circuit of the Debye model. The electrical circuit elements correspond to the physical dielectric susceptibility quantities as follows: the instantaneous dielectric constant $\varepsilon_{0}=C_{1}$ and the strength $\Delta \varepsilon=C_{2}$. The resistor $R$ represents the viscosity $\eta$. The relaxation time is given by $\tau_{D}=R C_{2}$.

$$
\widetilde{\varepsilon}(\omega)=\varepsilon_{\infty}+\frac{\Delta \varepsilon}{1+i \omega \tau_{D}},
$$

where $\tau_{D}$ is proportional to the viscosity $\eta$. In the highfrequency limit the capacitance of Eq. (1) is given by the capacitor $C_{1}$ alone. The capacitor $C_{1}$ is thus representing the high-frequency dielectric susceptibility $\varepsilon_{\infty}$, which is sometimes referred to as the "glassy" response of the liquid. ${ }^{43}$ The resistor on the other hand represents the viscosity of the surrounding liquid which "hinders" the rotation of the dipoles. The capacitor $C_{2}$ represents the strength of the dielectric relaxation $\Delta \varepsilon=\varepsilon_{0}-\varepsilon_{\infty}$ (which is directly related to the dipole density and the size of the molecular dipole moment).

To be able to model the asymmetric relaxation, which is not captured by the Debye model, we add a constant phase element CPE, as used by Jonscher and others, ${ }^{42,44-47}$ in parallel with the resistor (see Fig. 2). We place the CPE in parallel because our assumption is that the relaxation process, which is described by the CPE, is a relaxation of the same dipoles that relax in Debye-like manner at low frequencies. The CPE has a capacitance given by:

$$
\widetilde{C}_{\mathrm{CPE}}(\omega)=K(i \omega)^{-\alpha} \quad 0<\alpha<1,
$$

where $K$ has the dimension of capacitance $\times$ time $^{-\alpha}$. The $\mathrm{CPE}$ has its name because the complex capacitance of the CPE has a constant phase angle $[-(\pi / 2) \alpha]$ independent of the frequency. The limits $\alpha=0$ and $\alpha=1$ correspond to a capacitor and a resistor, respectively.

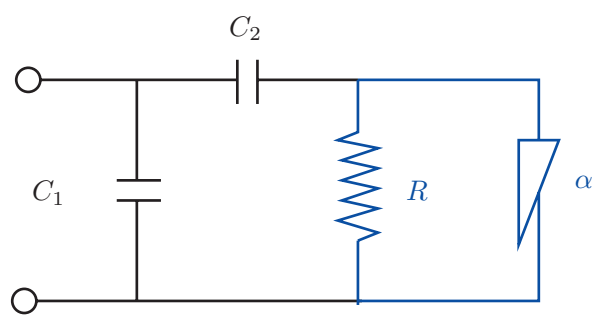

FIG. 2. The circuit model for alpha relaxation. The Debye model circuit is extended by adding a constant phase element (CPE) with a capacitance $\widetilde{C}_{\mathrm{CPE}}(\omega)=K(i \omega)^{-\alpha} 0<\alpha<1$. The CPE governs the high-frequency behavior of the response. $C_{1}$ corresponds to $\varepsilon_{\infty}$ and $C_{2}$ corresponds to $\Delta \varepsilon$ as in Fig. 1.

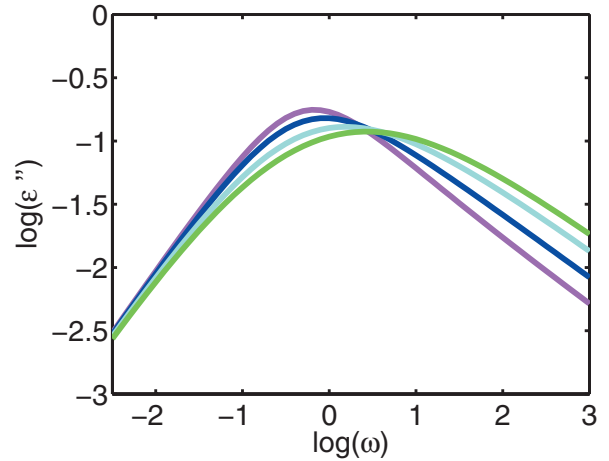

FIG. 3. The imaginary part of the circuit model for the alpha relaxation [Eq. (4)] with the exponent $\alpha=0.5$. The values of $k_{\alpha}$ are varied from 0.9 up to 3.5 corresponding to the most narrow and most broad spectral shape. (The dielectric strength, $\Delta \varepsilon$, and the time $\tau_{\alpha}$ are the same for all curves.)

The introduction of the CPE leads to our new function describing the dielectric alpha relaxation (with $k_{\alpha}=K \tau_{\alpha}^{\alpha} / \Delta \varepsilon$ and $\left.\tau_{\alpha}=\tau_{D}\right)$,

$$
\begin{aligned}
\widetilde{\varepsilon}(\omega) & =\varepsilon_{\infty}+\frac{1}{\frac{1}{\Delta \varepsilon}+\frac{1}{\frac{1}{i \omega R}+\frac{K}{(i \omega)^{\alpha}}}} \\
& =\varepsilon_{\infty}+\frac{\Delta \varepsilon}{1+\frac{1}{\left(i \omega \tau_{\alpha}\right)^{-1}+k_{\alpha}\left(i \omega \tau_{\alpha}\right)^{-\alpha}}} .
\end{aligned}
$$

An illustration of the imaginary part of this function is shown in Fig. 3.

\section{B. Physical interpretation of the alpha circuit model}

Equation (4) can be used as a fitting function, but the electrical circuit also serves as a model which gives the basis for an interpretation of the function. Replacing the resistor $R$, which corresponds to viscosity in the Debye model, with a resistor in parallel with a CPE can be viewed as replacing the static viscosity, $\eta$, with the dynamical frequency dependent viscosity $\tilde{\eta}(\omega)$. In this interpretation the model is close to earlier models which relate the mechanical response seen in viscosity and shear modulus to the dielectric response. In Ref. 48 such a model is constructed exactly by replacing the static viscosity of the Debye model by a dynamical viscosity. Equivalent models have earlier been developed starting from a microscopic perspective, first by Gemant, ${ }^{49}$ and later by DiMarzio and Bishop. ${ }^{50}$ A short review is given in Ref. 51 where it is also demonstrated that the connection between the mechanical relaxation and the dielectric relaxation are qualitatively described by this model, though the quantitative agreement is less convincing. In this interpretation the asymmetry of the dielectric relaxation is "inherited" from asymmetry in the mechanical relaxation, even if the spectral shape of the two is different (see Ref. 51 for a detailed discussion).

For completeness one could add an extra capacitor in parallel with the resistor and the CPE in Fig. 2. Such a capacitor would represent the high-frequency elastic response of the liquid and would have a capacitance proportional to the inverse of the high-frequency shear modulus. This elastic 


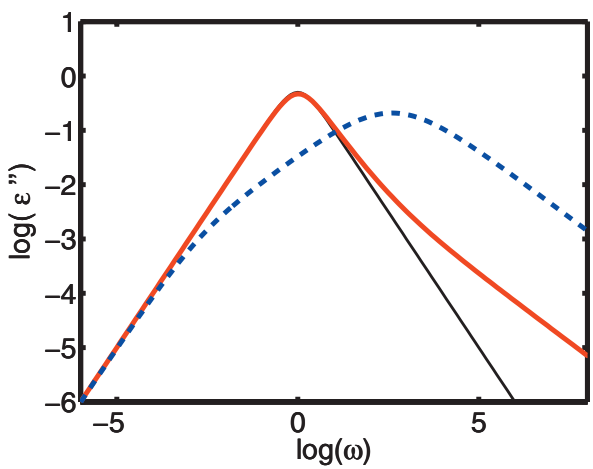

FIG. 4. The curves show the imaginary part of the circuit model all with the exponent $\alpha$ set to 0.5 . Two different $k_{\alpha}$-values are shown: $k=0.1$ (full line), and $k=20$ (dashed line). The thin line is the Debye relaxation. (The dielectric strength, $\Delta \varepsilon$, and the time, $\tau_{\alpha}$, is the same for all three curves.)

response can actually be seen in the dielectric response as demonstrated in Ref. 51. However, the spectral shapes that can be achieved do not change when adding this capacitance; to keep the model as simple as possible we have therefore left it out.

\section{The characteristics of the alpha circuit model}

The function in Eq. (4) has a total of five adjustable parameters: The strength, $\Delta \varepsilon$, the relaxation time, $\tau_{\alpha}$, the high-frequency level $\varepsilon_{\infty}$, and finally the two shape parameters $\alpha$ and $k_{\alpha}$. This means that the function has one additional parameter compared to the CD function and the KWW function. However, it still has the same low-frequency behavior with a power law of exponent 1 . The extra parameter, $k_{\alpha}$, gives the function a variable width for the same highfrequency exponent. This is illustrated in Fig. 3.

It can be seen both from the circuit and from the mathematical expression that this model in some sense can be regarded as a type of combination of the Debye model and the CC model-since leaving out the constant phase element gives a Debye model while leaving out the resistor gives a $\mathrm{CC}$ model $^{42}$ (see Sec. III). The Debye part dominates at low frequencies, whereas the $\mathrm{CC}$ part dominates at high frequencies. The parameter $k_{\alpha}$ determines where (with respect to the relaxation peak) this transition between the two behaviors takes place. For very small $k_{\alpha}$ values the transition comes significantly after the relaxation peak leading to an inflection point on the high-frequency side. Very large $k_{\alpha}$ values will on the other hand make the CC-like behavior dominate down to below the peak. These behaviors of the model are illustrated in Fig. 4. The values of $k_{\alpha}$ that give well-behaved peaks depend on the value of alpha. For $\alpha=0.5$, which is our focus, we find that $k_{\alpha}$ should be chosen in the range of 0.9 3.5; the range of widths obtained is illustrated in Fig. 3.

We also find that the circuit model can give spectral shapes with good correspondence to both KWW and CD functions. There is not a one to one correspondence between the circuit model and the CD function or the circuit model and the KWW, but a reasonably good overlap can be obtained. The exact parameters found when fitting the circuit model to the CD or the KWW function depend significantly on how large a frequency range is included.

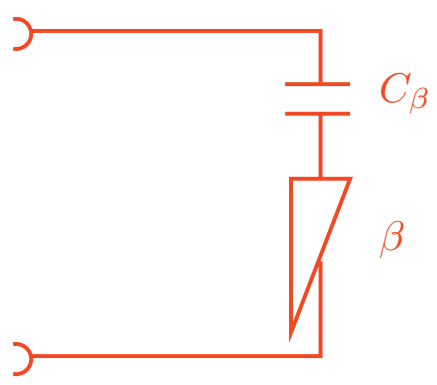

FIG. 5. Part of the circuit model for a Cole-Cole function [to obtain a full Cole-Cole model (Ref. 42) this part is connected in parallel with the capacitor $C_{1}$ ]. The constant phase element $(\mathrm{CPE})$ is with a capacitance $\widetilde{C}_{\mathrm{CPE}}(\omega)$ $=K(i \omega)^{-\beta}, 0<\beta<1$ and is used to model the beta process. $C_{\beta}$ represents $\varepsilon_{\beta}$.

\section{BETA RELAXATION}

Before building the merging model, we take a look at the phenomenology of the secondary beta process. Generally it is observed that all liquids have one or more beta relaxations. The beta relaxation can give rise to a well-resolved highfrequency peak but it can also sometimes manifest itself as an excess wing on the high-frequency side of the alpha relaxation. ${ }^{53-55}$ An evolution from excess wing into a secondary peak has been seen in long-time annealing experiments $^{53,56-59}$ as well as in high-pressure dielectric experiments (see, e.g., Ref. 7).

Regarding the temperature dependence of the beta relaxation in the equilibrium liquid, it is found that the relaxation time is temperature independent or very weakly temperature dependent, while the amplitude of the beta relaxation generally increases with temperature. In the glass, the beta process slows down in an Arrhenius manner but the amplitude is more or less constant with cooling. ${ }^{60-62}$

In all cases the beta relaxations' spectral shape is seen to be symmetric, relatively broad, and temperature independent (see, e.g., Ref. 6). Thus, the CC function gives a good description of this process and is almost always used to fit it. Cole and Cole illustrated their function ${ }^{42}$ by the electrical circuit shown in Fig. 5; a capacitor in series with a $\mathrm{CPE}_{\beta}$ with a slope parameter $\beta$. This leads to the well-known expression

$$
\widetilde{\varepsilon}_{\beta}(\omega)=\frac{1}{\frac{1}{\varepsilon_{\beta}}+\frac{(i \omega)^{\beta}}{K}}=\frac{\varepsilon_{\beta}}{1+\left(i \omega \tau_{\beta}\right)^{\beta}}
$$

for the CC function, where $\varepsilon_{\beta}$ corresponds to the capacitor in the circuit, and $\tau_{\beta}=\left(\varepsilon_{\beta} / K\right)^{1 / \beta}$.

\section{THE MERGING OF THE ALPHA AND BETA RELAXATIONS}

Having established a model for the alpha relaxation that fulfills our requirements and a model for the beta relaxation, we now move on to combining the two in a model of the alpha-beta merging.

When combining the $\mathrm{CC}$ model with the previously described alpha model, there are two simple possibilities. The CC model can be attached at the node between $C_{1}$ and $C_{2}$, 


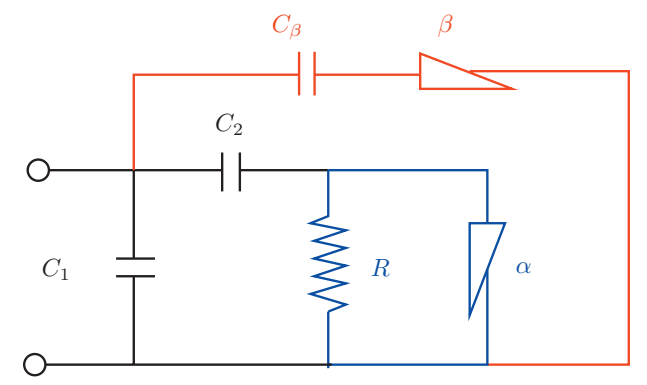

(a)

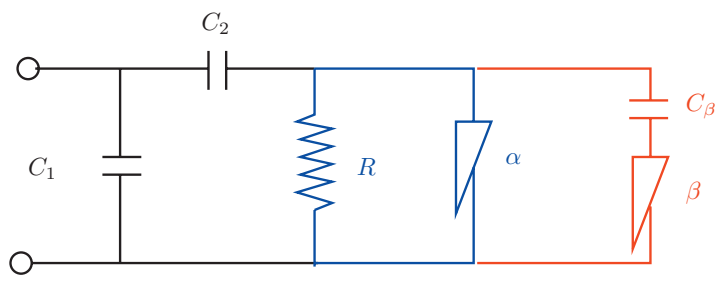

(b)

FIG. 6. The electrical circuit of (a) the additive model and (b) the alpha-beta merging circuit model. $C_{1}$ corresponds to $\varepsilon_{\infty}$ and $C_{2}$ corresponds to $\Delta \varepsilon$ as in Fig. 1. The respective mathematical expressions are Eqs. (6) and (7).

thus being in parallel with every element in the model, or it can be attached in parallel with the two alpha elements $R$ and $\mathrm{CPE}_{\alpha}$. This is illustrated in Fig. 6.

These two possibilities lead us to two different modelswhich also have different physical interpretations. In the first case an extra branch is added to the circuit [Fig. 6(a)] and there is a new place for loading charge, namely, $C_{\beta}$. In the dielectric case it means that the total dipole relaxation becomes larger when introducing the beta relaxation. In the second case the beta relaxation gives a new mechanism for relaxation, but the amount of charge in the low-frequency limit is unchanged. In the dielectric case this means that the beta relaxation has no effect on the total strength of the relaxation. The dipoles relaxing are the same with or without the beta relaxation; it is the manner in which they relax which is perturbed when introducing the beta relaxation.

If we determine the response by calculating the capacitance of the two models, we find that the first option results into the well-known simple addition of the alpha and beta models (henceforth this model is referred to as the additive model):

$$
\begin{aligned}
& \widetilde{\varepsilon}^{A}(\omega)=\varepsilon_{\infty}+\frac{1}{\frac{1}{\Delta \varepsilon}+\frac{1}{\frac{1}{i \omega R}+\frac{1}{K_{\alpha}(i \omega)^{\alpha}}}}+\frac{1}{\frac{1}{\varepsilon_{\beta}}+K_{\beta}(i \omega)^{\beta}} \Leftrightarrow \\
& \widetilde{\varepsilon}^{A}(\omega)=\varepsilon_{\infty}+\frac{\Delta \varepsilon}{1+\frac{1}{\left(i \omega \tau_{\alpha}\right)^{-1}+k_{\alpha}\left(i \omega \tau_{\alpha}\right)^{-\alpha}}}+\frac{\varepsilon_{\beta}}{1+\left(i \omega \tau_{\beta}\right)^{\beta}} .
\end{aligned}
$$

The second possibility leads to a new way of combining the alpha and the beta models. We will refer to it as the alphabeta circuit model:
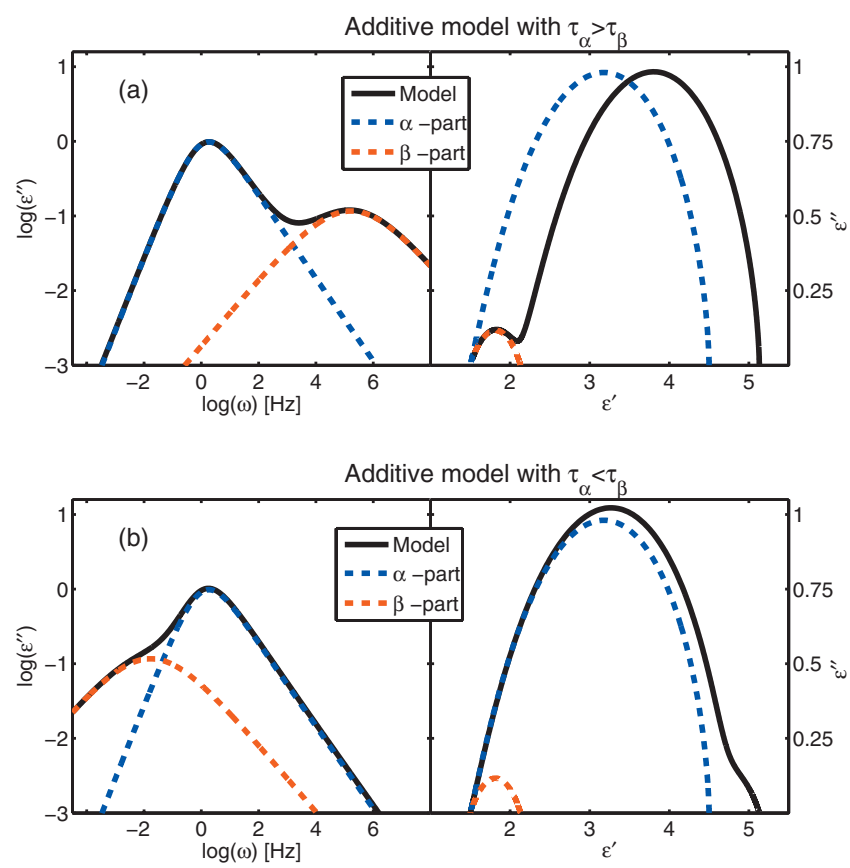

FIG. 7. Spectra for the additive model with an alpha relaxation time $\tau_{\alpha}$ $=1 \mathrm{~s}$ and two different beta relaxation times. In (a) $\tau_{\beta}=10^{-5} \mathrm{~s}$ and in (b) $\tau_{\beta}=10^{2} \mathrm{~s}$. (The rest of the parameters have following values: $\varepsilon_{\infty}=1.5, \Delta \varepsilon$ $=3, k_{\alpha}=1, \alpha=0.55, \varepsilon_{\beta}=0.8$, and $\beta=0.45$.)

$$
\begin{aligned}
& \widetilde{\varepsilon}^{C}(\omega)=\varepsilon_{\infty}+\frac{1}{\frac{1}{\Delta \varepsilon}+\frac{1}{\frac{1}{i \omega R}+\frac{1}{K_{\alpha}(i \omega)^{\alpha}}+\frac{1}{\frac{1}{\varepsilon_{\beta}}+K_{\beta}(i \omega)^{\beta}}}} \\
& \widetilde{\varepsilon}^{C}(\omega)=\varepsilon_{\infty}+\frac{\Delta \varepsilon}{1+\frac{1}{\left(i \omega \tau_{\alpha}\right)^{-1}+k_{\alpha}\left(i \omega \tau_{\alpha}\right)^{-\alpha}+\frac{\varepsilon_{\beta} / \Delta \varepsilon}{1+\left(i \omega \tau_{\beta}\right)^{\beta}}}},
\end{aligned}
$$

with $\tau_{\beta}=\left(\varepsilon_{\beta} K_{\beta}\right)^{1 / \beta}$. Equation (7) is the main new result of this paper. Spectra of the two models are illustrated in Figs. 7 and 8.

Both models involve 8 parameters: There are two shape parameters for the alpha relaxation $\left(k_{\alpha}\right.$ and $\left.\alpha\right)$, one shape parameter for the beta relaxation $(\beta)$, a characteristic time for each process $\left(\tau_{\alpha}\right.$ and $\left.\tau_{\beta}\right)$, a strength parameter for each process $\left(\Delta \varepsilon\right.$ and $\left.\varepsilon_{\beta}\right)$ and the high-frequency dielectric constant $\left(\varepsilon_{\infty}\right)$.

The two models result in very similar spectra when the beta relaxation is faster than the alpha relaxation. The beta relaxation gives rise to either a peak, a shoulder, a wing or just an apparent broadening of the alpha relaxation. This means that high-frequency slopes of the alpha relaxation can appear to be numerically smaller than $1 / 2$ due to an underlying beta relaxation. On the other hand it is not possible to explain high-frequency slopes numerically larger than $1 / 2$ by the influence of the beta relaxation. Even though the models appear similar they differ because of the fundamental difference in the way the beta relaxation is introduced in the circuit as discussed above. This difference also leads to differ- 

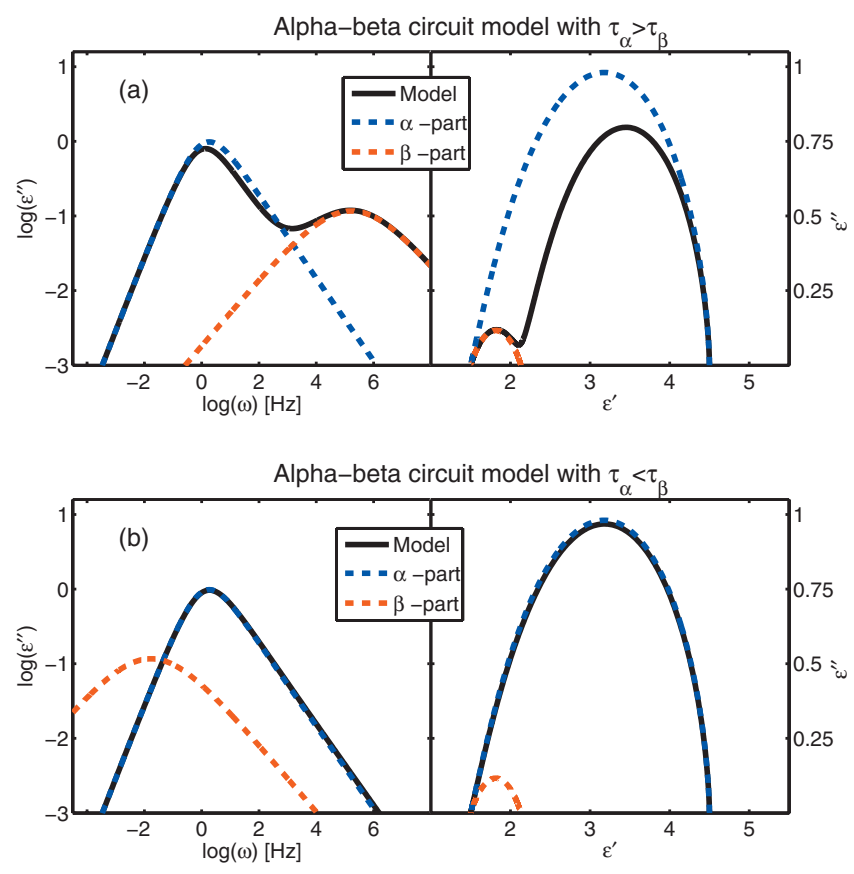

FIG. 8. Spectra for the alpha-beta merging circuit model with an alpha relaxation time $\tau_{\alpha}=1 \mathrm{~s}$ and two different beta relaxation times. In a) $\tau_{\beta}$ $=10^{-5} \mathrm{~s}$ and in b) $\tau_{\beta}=10^{2} \mathrm{~s}$. (The rest of the parameters have following values: $\varepsilon_{\infty}=1.5, \Delta \varepsilon=3, k_{\alpha}=1, \alpha=0.55, \varepsilon_{\beta}=0.8$, and $\beta=0.45$.)

ences in the spectra-especially for some choices of the parameters. In the following section we discuss the differences in terms of how the spectra look with different values of the parameters in the model and show why the circuit model, in our opinion, is more appropriate for describing the merging of the alpha relaxation with the Johari-Goldstein beta relaxation.

\section{A. The characteristics of the two alpha-beta models}

In the circuit model the beta relaxation is not an individual relaxation, but a part of the alpha relaxation. When comparing the behavior of the circuit model with the additive model we want to follow the underlying models for the "pure relaxations." We define these pure relaxations as the limits of the full model when one relaxation is much slower than the other. Thus, the pure alpha relaxation is modeled with the limit of the total relaxation when $\tau_{\beta} \rightarrow \infty$. With this definition the pure alpha model corresponds exactly to the alpha model as it looked before the beta process was introduced. The pure beta relaxation model is likewise defined as the limit of the total model when the alpha relaxation is much slower than the beta relaxation, $\tau_{\alpha} \rightarrow \infty$. This leads to the following underlying pure models for the circuit model:

$\widetilde{\varepsilon}_{\alpha}^{C}(\omega)=\lim _{\tau_{\beta} \rightarrow \infty} \widetilde{\varepsilon}(\omega)=\varepsilon_{\infty}+\frac{\Delta \varepsilon}{1+\frac{1}{\left(i \omega \tau_{\alpha}\right)^{-1}+k_{\alpha}\left(i \omega \tau_{\alpha}\right)^{-\alpha}}}$,

$\widetilde{\varepsilon}_{\beta}^{C}(\omega)=\lim _{\tau_{\alpha} \rightarrow \infty} \widetilde{\varepsilon}(\omega)=\varepsilon_{\infty}+\frac{\varepsilon_{\beta} \Delta \varepsilon}{\varepsilon_{\beta}+\Delta \varepsilon\left(1+\left(i \omega \tau_{\beta}\right)^{\beta}\right)}$,

and trivially for the additive model:

$$
\begin{aligned}
& \widetilde{\varepsilon}_{\alpha}^{A}(\omega)=\lim _{\tau_{\beta} \rightarrow \infty} \widetilde{\varepsilon}(\omega)=\varepsilon_{\infty}+\frac{\Delta \varepsilon}{1+\frac{1}{\left(i \omega \tau_{\alpha}\right)^{-1}+k_{\alpha}\left(i \omega \tau_{\alpha}\right)^{-\alpha}}}, \\
& \widetilde{\varepsilon}_{\beta}^{A}(\omega)=\lim _{\tau_{\alpha} \rightarrow \infty} \widetilde{\varepsilon}(\omega)=\varepsilon_{\infty}+\frac{\varepsilon_{\beta}}{1+\left(i \omega \tau_{\beta}\right)^{\beta}} .
\end{aligned}
$$

The two main features in which the models differ are (i) the behavior at low frequencies and (ii) the interpretation of how the two relaxation processes affect the total relaxation strength as can be seen in Figs. 7 and 8 .

\section{Low-frequency behavior}

The additive model sums the two relaxations, therefore the low-frequency limit will have a $\omega^{\beta}$ dependence (since $\beta<1$ ). The beta relaxation will in other words be seen as a wing or a shoulder on the low-frequency side of the alpha relaxation when the alpha relaxation is slower than the beta relaxation. This does not have much practical effect for fitting data, because this wing signal will be extremely small. However, if the beta relaxation is slower than the alpha relaxation it will pop out as a low-frequency shoulder, as illustrated in Fig. 7(b).

The alpha-beta circuit model shows Debye-like behavior in the low-frequency range, and it always has a $\omega^{1}$-behavior in the low-frequency limit. Or put in other words: The beta relaxation is not seen on the low-frequency side of the alpha relaxation. This means that the beta relaxation truly merges with the alpha relaxation when it becomes slower. This is illustrated with a $\log$ - $\log$ plot of $\varepsilon^{\prime \prime}$ as a function of the frequency $\omega$ in Fig. 8(b).

\section{Relation between the relaxation strengths}

The relation between the amplitudes or strength of the relaxation processes is clearly illustrated in the Nyquist plots. In the additive model the total relaxation strength increases when there is a beta relaxation, compared to the relaxation strength of the alpha relaxation. In the alpha-beta circuit model on the other hand, the total relaxation strength is given by the strength of the alpha relaxation alone. This means that introducing the beta relaxation does not add something extra; rather the beta relaxation makes the alpha relaxation bifurcate in two. It has the consequence that the apparent strength of the alpha relaxation is smaller than the strength it would have if there were no beta relaxation. This is shown in Fig. 7(a) for the additive model and Fig. 8(a) for the alpha-beta circuit model. Note that the actual strength of the beta relaxation in the alpha-beta circuit model is given by $\Delta \varepsilon_{\beta}$ $=\varepsilon_{\beta} \Delta \varepsilon /\left(\varepsilon_{\beta}+\Delta \varepsilon\right)$, meaning that it is close to $\varepsilon_{\beta}$ as long as the latter is much smaller than the total strength $\Delta \varepsilon$. This more complicated relation between the beta amplitude parameter is a consequence of the way the model interlinks the two processes.

\section{Relation to Williams ansatz}

In this section we will briefly compare the alpha-beta circuit model to the Williams ansatz. The Williams ansatz is naturally formulated in the time domain in terms of the cor- 
relation function, $\phi(t)=\phi_{\alpha}(t)\left((1-\lambda)+\lambda \phi_{\beta}(t)\right), 0<\lambda<1$. From this expression it is easily seen that the beta relaxation only has an effect before the decay of the alpha relaxation. This means that the low-frequency behavior will be that of the alpha relaxation and that the beta relaxation will not be visible if it is faster than the alpha relaxation. Thus the qualitative behavior of the alpha-beta circuit model is equivalent to the behavior obtained when using the Williams ansatz at low frequencies. The Williams ansatz approach does not give any clear answers regarding the influence that the relaxation strength of the beta relaxation has on the total relaxation strength. This is because the alpha and beta relaxation as well as the total relaxation all are presented in a normalized form.

\section{B. Physical interpretation of the two alpha-beta models}

The two models reflect two different physical interpretations of the origin of the beta relaxation. In the additive model only the alpha process is related to the relaxation of the dipole orientation while the beta process is completely decoupled from the alpha process. The beta process continues to exist even when it becomes slower than the alpha relaxation. Interestingly in this respect, it was recently found by Mandanici et al. ${ }^{63}$ using mechanical spectroscopy that ethylcyclohexane has an intramolecular motion (a "chair" bending mode) which is seen in the relaxation spectra as a secondary process which has a continuous temperature dependence seen on both sides of the alpha relaxation. We believe that the additive model would be suited for fitting data containing an extra process due to this type of internal degrees of freedom.

In contrast, in the alpha-beta circuit model the beta relaxation is an integrated part of the total relaxation process, and the merging region becomes one where, upon cooling, the total relaxation process separates in two processes. Phenomenologically we know that the beta relaxation is weakly temperature dependent in the equilibrium liquid, ${ }^{60,61}$ while the alpha relaxation is strongly temperature dependent. When the liquid is heated, the alpha relaxation passes the timescale of the beta relaxation "picking it up" and merging into one single integrated process. This integrated process has the spectral features of the alpha relaxation. Both the alpha and the beta process are a relaxation of the dipole represented by $\Delta \varepsilon$. This interpretation is consistent with the view that the alpha and beta relaxation are closely related. It is clearly consistent with the idea of the two processes being, respectively, large and small angle motions of the same dipole. The phenomenology of the circuit model agrees well with the phenomenology of the Johari-Goldstein beta process since it does not appear on the low-frequency side of the alpha relaxation. We therefore believe that this model is a good candidate when fitting spectra including an alpha and a beta relaxation. Diezemann and co-workers in $1999^{64}$ discussed a stochastic free-energy landscape model in which the beta relaxation corresponds to intraminima transitions. In this model, as in the alpha-beta circuit model proposed here, it is not possible to have the timescales of alpha and beta relaxations cross each other such that the beta process becomes slower than the alpha process.

\section{FITTING TO DATA}

\section{A. Data}

We have fitted the alpha-beta circuit model to dielectric data from two liquids: the dielectric measurements that are used in this paper are made on the liquids di-iso-butyl phthalate (99\%, Sigma-Aldrich Chemical Co., DisoBP) and Toluene-Pyridine mixture (17\% toluene, tol-pyr). The data have already been published in Refs. 8 and 16, respectively. The tol-pyr data were obtained in a setup described in details in Refs. 65 and 66, and the DisoBP data were obtained in a setup described in Ref. 67-69. The two liquids frequency scans are characterized by well-revealed secondary processes in the megahertz range.

\section{B. Fitting procedure}

The alpha-beta circuit model has a total of eight parameters, five to describe the alpha relaxation and three to describe the beta relaxation. (Recall that there are only two shape parameters for the alpha relaxation and one shape parameter for the beta relaxation, the additional parameters are time scales and relaxation strength). This is the same number of parameters as in the additive model, and also the same number of parameters as one would have with an additive model of a $\mathrm{HN}$-function and a CC. It is not possible to uniquely determine these eight parameters in the region where the alpha and beta process merge. There are simply too few distinct features in the spectra. In order to get a stable fit it is therefore necessary to make some reasonable assumptions.

As described in the introduction we believe ${ }^{8}$ that the alpha relaxation broadens upon cooling until the numerical

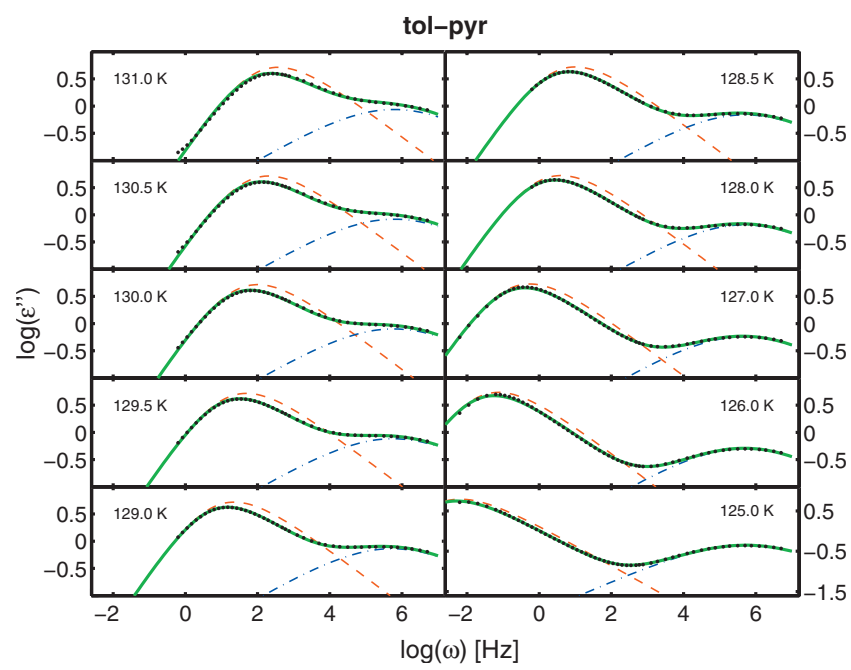

FIG. 9. The results from fitting the alpha-beta circuit model to tol-pyr data. The three shape parameters are held fixed at all temperatures implying TTS for the individual alpha and beta process, respectively $\left(k_{\alpha}=1.8, \alpha=0.5\right.$, and $\beta=0.39)$. The dots show the data. The full line is the total fit. The dashed line is the pure alpha relaxation and the dashed-dotted line is the pure beta relaxation (both defined in Sec. IV A 1). 


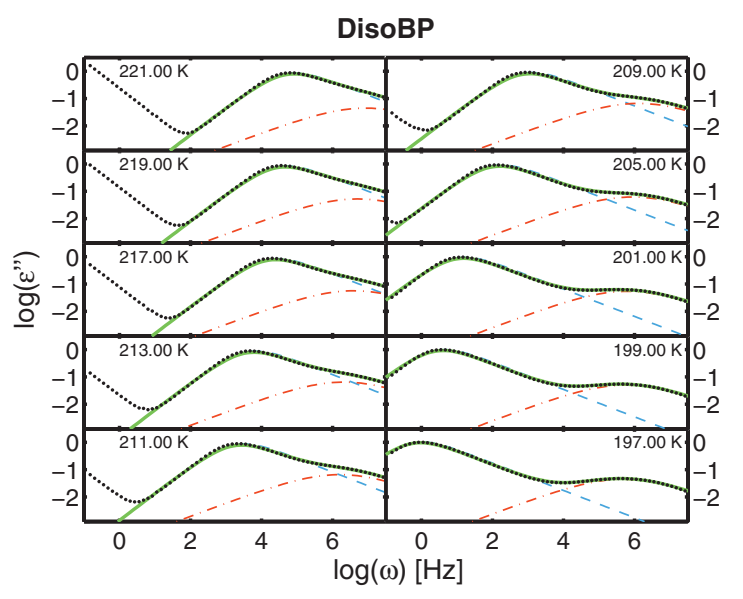

FIG. 10. The results from fitting the alpha-beta circuit model to DisoBP data. The three shape parameters are held fixed at all temperatures implying TTS for the individual alpha and beta process, respectively $\left(k_{\alpha}=0.93, \alpha\right.$ $=0.5$, and $\beta=0.49$ ). The full line is the total fit. The dashed line is the pure alpha relaxation and the dashed-dotted line is the pure beta relaxation (both defined in Sec. IV A 1).

value of the slope reaches $1 / 2$ upon further cooling we believe that the alpha relaxation obeys TTS. This is based on our earlier results, ${ }^{8}$ where a study on 347 spectra for 53 liquids show prevalence of high-frequency slopes with the numerical value close to $1 / 2$. Slopes numerically smaller than $1 / 2$ are explained by the influence of the beta relaxation, and it is this last point that we study closer in this work. To do so we make fits of the spectra in the merging region assuming that the alpha and beta processes both obey TTS,
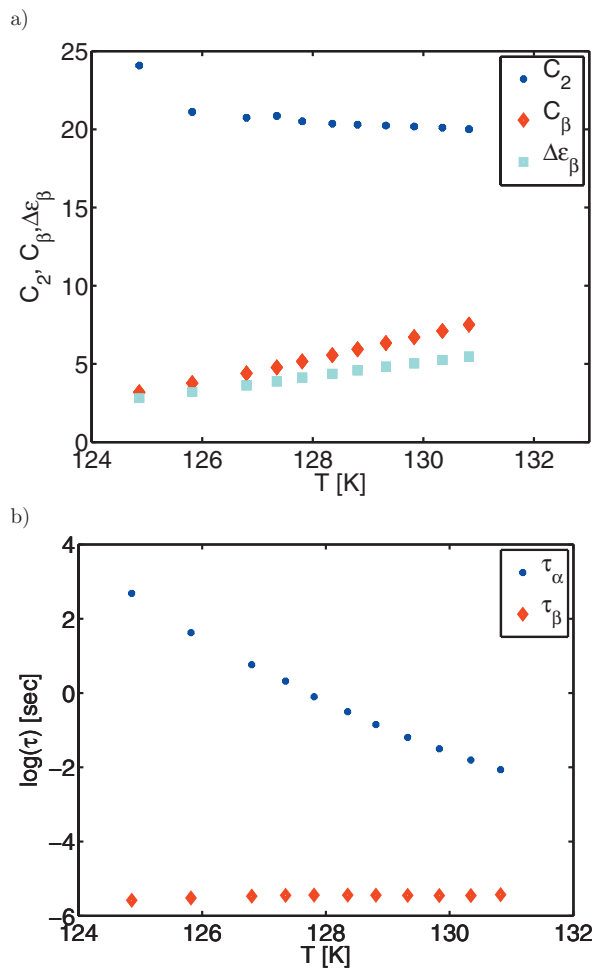

FIG. 11. (a) Fitted amplitudes found for tol-pyr. $C_{2}=\Delta \varepsilon$ is the total relaxation strength, $C_{\beta}=\varepsilon_{\beta}$ is the parameter controlling the beta relaxation amplitude. $\Delta \varepsilon_{\beta}$ is the actual strength of the beta relaxation as it merges with the alpha relaxation. (b) The temperature dependence of the fitted relaxation times - blue for alpha and red for beta relaxation-found for tol-pyr.
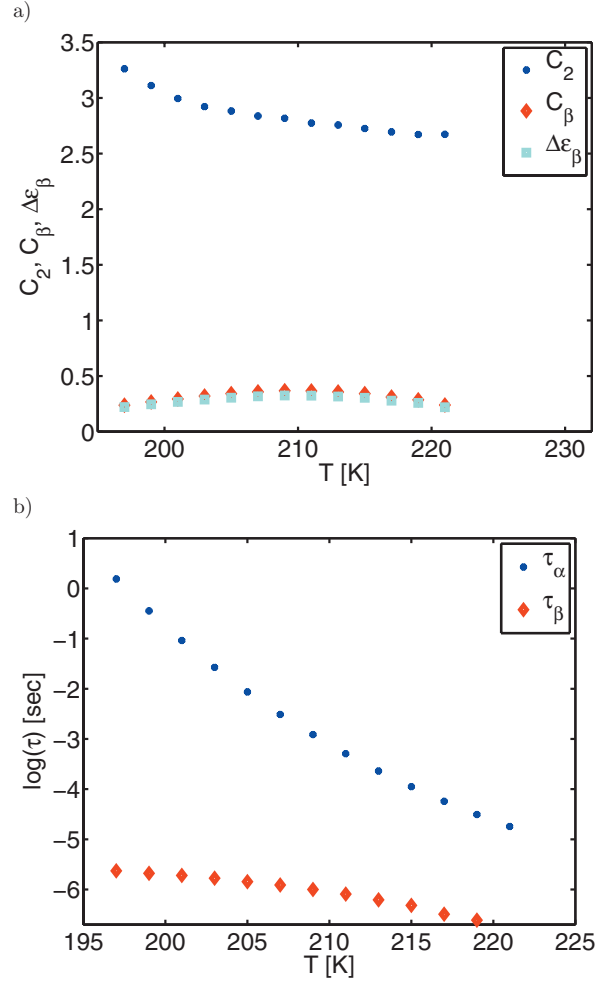

FIG. 12. (a) Fitted amplitudes found for DisoBP. $C_{2}=\Delta \varepsilon$ is the total amplitude, $C_{\beta}=\varepsilon_{\beta}$ is the parameter controlling the beta relaxation amplitude. $\Delta \varepsilon_{\beta}$ is the actual amplitude of the beta relaxation as it merges with the alpha relaxation. (b) Fitted relaxation times found for DisoBP.

and that the numerical value of the high-frequency slope of the alpha relaxation is $1 / 2$. In the fitting procedure this means letting the three shape parameters $k_{\alpha}, \alpha$, and $\beta$ be temperature independent, and specifically setting $\alpha=1 / 2$. The values for $k_{\alpha}$ and $\beta$ were determined by making preliminary fits at temperatures where the alpha and beta peaks are wellseparated. With three parameters fixed we have five remaining fitting parameters, of which $\varepsilon_{\infty}$ is a pure real parameter which just gives the high-frequency level of the dielectric constant. The remaining four parameters are the two time constants and the two amplitude parameters.

\section{The results of the fits}

The results from the model fit for tol-pyr $\left(k_{\alpha}=1.8\right.$, $\alpha=0.5$, and $\beta=0.39)$ are presented in Fig. 9 and the DisoBP data and fits $\left(k_{\alpha}=0.93, \alpha=0.5\right.$, and $\left.\beta=0.49\right)$ are shown in Fig. 10. We find a good correspondence between the data and the fit, especially in the region where we see the merging of the alpha and the beta peak. For DisoBP there is a systematic discrepancy just on the low-frequency side of the alpha peak. A better fit around the peak can be obtained using a lower value of $k_{\alpha}=0.65$; however this gives rise to a highfrequency inflection point in the pure alpha relaxation. This inflection point is difficult to see directly in the fit but comes out in the derivative (this feature of the alpha model was discussed in Sec. II C). Because we are interested in the high-frequency slope we prefer a small discrepancy around the peak. However, it does show that the alpha model does not fully capture the spectral shape of the alpha relaxation. 


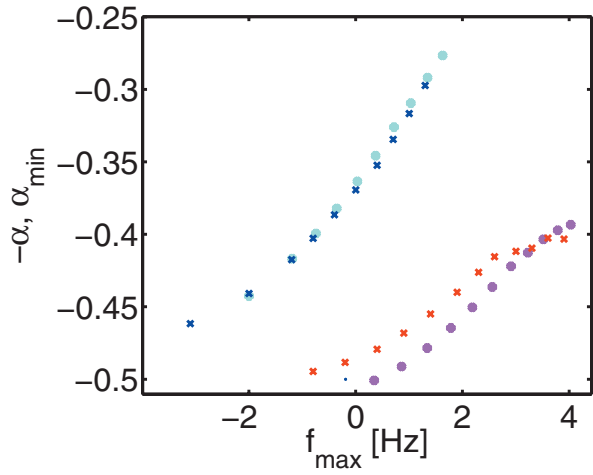

FIG. 13. Minimum slopes defined as in Ref. 8 shown as a function of the alpha relaxation loss peak frequency. Light blue circles are tol-pyr data, blue crosses are tol-pyr fit, magenta circles are DisoBP data, and red crosses are DisoBP fit.

Tol-pyr has a relatively strong beta process and some of the characteristics of the circuit model are therefore seen more clearly here: The beta relaxation "eats" a bit of the alpha relaxation, so that the amplitude at the peak is smaller than the amplitude of the "pure" alpha relaxation. Also, the beta strength differs from $\varepsilon_{\beta}$, and is instead given by $\Delta \varepsilon_{\beta}$ $=\varepsilon_{\beta} \Delta \varepsilon /\left(\varepsilon_{\beta}+\Delta \varepsilon\right)$. From the fitting parameters we find the well-known dramatic increase in the alpha relaxation time as temperature decreases, while the beta relaxation time is very weakly temperature dependent in the equilibrium liquid. ${ }^{60-62}$ See Fig. 11(a) (for tol-pyr) and Fig. 12(a) (for DisoBP). The alpha relaxation strength increases with decreasing temperature as expected in dielectric relaxation. The beta relaxation amplitude has a decreasing trend with decreasing temperature for tol-pyr [Fig. 11(b)] and is more or less temperature independent for DisoBP [Fig. 12(b)].

Regarding the shape parameters, the good quality of the fits, especially on the high-frequency side of the alpha peak, confirms that for these liquids it is possible to explain highfrequency slopes numerically smaller than $1 / 2$, by the influence of the beta relaxation. In Fig. 13 we show the phenomenological minimum slopes found for DisoBP and tol-pyr in Ref. 8. These approach the numerical value of $1 / 2$ when temperature decreases. We moreover show the minimum slopes of the curve obtained from the fits. In the fits we have used a high-frequency slope numerically equal to $1 / 2$ for the alpha relaxation at all temperatures. The deviation from this value in the total spectra in the fit is therefore due to the influence of the beta relaxation. The correspondence between the minimum slopes of the tol-pyr data and fit is good. For DisoBP there is also a good qualitative agreement. This supports the view that alpha relaxation high-frequency slopes that are found to be numerically smaller than $1 / 2$ can be attributed to the influence of the beta relaxation. Slopes that are numerically larger than $1 / 2$ cannot be explained in this way. As pointed out earlier, we believe that these numerically larger slopes (and narrower peaks) appear as the liquid is heated and approaches the Debye behavior at high temperatures. When slopes numerically larger than $1 / 2$ are observed even at low temperatures (with low loss peak frequency) we conjecture that this is because one is still above the tempera-

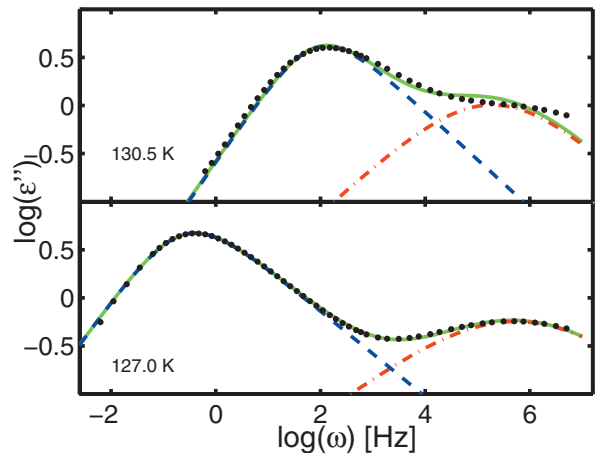

FIG. 14. The result from fitting a sum of a $\mathrm{HN}$-function and a $\mathrm{CC}$ function to tol-pyr data. The high-frequency slope is fixed to $1 / 2$, the two remaining shape parameters are fitted at the low temperature and held fixed at the high temperature implying TTS for the individual alpha and beta process, respectively $\left(\gamma_{\mathrm{HN}}=0.80, \beta_{\mathrm{HN}}=0.5 / \gamma_{\mathrm{HN}}=0.63\right.$, and $\left.\beta_{\mathrm{CC}}=0.50\right)$. The green dots show the data. The full line is the total fit. The dashed line is the pure alpha relaxation and the dashed-dotted line is the pure beta relaxation.

ture where the alpha and beta process merge, i.e., there is one or more low-lying beta processes.

\section{Comparison to a standard fit}

In this section we briefly compare our fits to what is probably the most commonly used fitting function used to describe the alpha-beta merging in dielectric spectra; a sum of a HN-function and a CC-function. This is illustrated with two temperatures of the tol-pyr data, a low temperature where the processes are well-separated and a high temperature where the processes are highly merged. In order to make the most direct comparison we use the same procedure as we did in the fit using the alpha-beta circuit model. The highfrequency power of the alpha relaxation is fixed to $1 / 2$, and the low temperature spectra is then fitted. TTS is assumed for each of the individual processes and the high temperature spectra are therefore fitted by adjusting only the time scales and the amplitudes. The result is shown in Fig. 14. The figure shows that the low temperature fit is good. The HN-function has the intrinsic problem (which is discussed in Sec. II) that the bluntness of the peak is obtained by letting the lowfrequency power be lower than the expected value 1 , in this case it is fitted to 0.8. Nevertheless the quality of the fit in the studied region is good for the low temperature spectra. The fit at higher temperature captures the overall features of the spectra but the overlap is rather poor in the details. This does not mean that the high temperature cannot be fitted with a sum of a HN-function and a CC function, it just means that more parameters need to be fitted than what we needed when using the alpha-beta circuit model. Either, one should allow the spectral shapes of each peak to be temperature dependent, or one should make a fit without the assumption of the $1 / 2$ high-frequency slope. The fact that the current fit does not work, while the fit in Fig. 9 made with the same assumptions and a different fitting function does work, clearly shows that the choice of fitting function influences the conclusions we draw. Comparing Figs. 9 and 14 it is also seen that the amplitudes of what we interpret as the pure relaxations is different, again showing how the fit used will influence the analysis of the problem. The alpha-beta circuit model is more 
satisfactory because it assures the correct low-frequency behavior and because the beta process can never be slower than the alpha process. We therefore believe that the conclusions drawn on the basis of this new model are more likely to be reliable.

\section{SUMMARY AND CONCLUSION}

We have presented a new circuit model that can be used to describe dielectric relaxation data. The circuit model leads to a fitting procedure for the combined alpha and beta relaxation. The connection to physics lies in the way that we combine the electrical circuit models for the alpha and the beta process, respectively, to describe the merging of the two processes. The model leads to an interpretation of the merging region as a type of bifurcation where the beta relaxation separates from the total relaxation, while it is still fundamentally related to the same physics as the alpha relaxation. One signature of this close interlink between the two processes in the model is that the beta relaxation does not add to the total amplitude of the relaxation.

The function used to fit the alpha relaxation is also new. This model has two shape parameters, one controlling the exponent of the high-frequency power law and one which independently controls the width of the relaxation. The lowfrequency behavior is always a Debye-like power law with exponent one, in agreement with dielectric relaxation data of molecular liquids.

The fact that the model is based on an electrical circuit ensures that it is physically consistent, e.g., obeys conservation of energy, positive dissipation, causality, linearity, etc. It moreover leads to an explicit function, with both real and imaginary parts, in the frequency domain which makes it convenient for fitting dielectric data.

The full alpha-beta circuit model was fitted to the merging region of two different molecular liquids. These fits were performed under the assumption that the intrinsic highfrequency behavior of the alpha relaxation is a $-1 / 2$ power law and that both the alpha and beta process separately obey TTS. We obtain good quality fits in the entire frequency and temperature range studied. This supports the view that highfrequency slopes numerically smaller than $1 / 2$ can be attributed to the influence of the beta relaxation.

\section{ACKNOWLEDGMENTS}

The authors thank Nick Bailey and Bo Jakobsen for useful comments. This work was supported by the Danish National Research Foundation's DNRF center for viscous liquid dynamics "Glass and Time."

${ }^{1}$ G. P. Johari and M. Goldstein, J. Chem. Phys. 53, 2372 (1970).

${ }^{2}$ G. P. Johari and M. Goldstein, J. Chem. Phys. 55, 4245 (1971).

${ }^{3}$ C. A. Angell, J. Non-Cryst. Solids 13, 133 (1991).

${ }^{4}$ T. Hecksher, A. I. Nielsen, N. B. Olsen, and J. C. Dyre, Nat. Phys. 4, 737 (2008).

${ }^{5}$ G. B. McKenna, Nat. Phys. 4, 673 (2008).

${ }^{6}$ L.-M. Wang and R. Richert, Phys. Rev. B 76, 064201 (2007).

${ }^{7}$ S. Capaccioli, K. Kessairi, D. Prevosto, M. Lucchesi, and P. A. Rolla, J. Phys.: Cond. Matter, 19, 064201 (2007).

${ }^{8}$ A. I. Nielsen, T. Christensen, B. Jakobsen, K. Niss, N. B. Olsen, R. Richert, and J. C. Dyre, J. Chem. Phys. 130, 154508 (2009).
${ }^{9}$ R. Böhmer, K. L. Ngai, C. A. Angell, and D. J. Plazek, J. Chem. Phys. 99, 4201 (1993).

${ }^{10}$ D. H. Huang and G. B. McKenna, J. Chem. Phys. 114, 5621 (2001).

${ }^{11}$ T. Scopigno, G. Ruocco, F. Sette, and G. Monaco, Science 302, 849 (2003).

${ }^{12}$ N. L. Ellegaard, T. Christensen, P. V. Christiansen, N. B. Olsen, U. R. Pedersen, T. B. Schrøder, and J. C. Dyre, J. Chem. Phys. 126, 074502 (2007).

${ }^{13}$ R. Böhmer, G. Diezemann, B. Geil, G. Hinze, A. Nowaczyk, and M. Winterlich, Phys. Rev. Lett. 97, 135701 (2006).

${ }^{14}$ K. L. Ngai and M. Paluch, J. Chem. Phys. 120, 857 (2004).

${ }^{15}$ R. Böhmer, G. Diezemann, G. Hinze, and E. Rössler, Prog. Nucl. Magn. Reson. Spectrosc. 39, 191 (2001).

${ }^{16}$ N. B. Olsen, T. Christensen, and J. C. Dyre, Phys. Rev. Lett. 86, 1271 (2001).

${ }^{17}$ A. Kudlik, C. Tschirwitz, T. Blochowicz, S. Benkhof, and E. Rössler, J. Non-Cryst. Solids 235-237, 406 (1998).

${ }^{18}$ G. Williams, in Dielectric and Related Molecular Processes, edited by M. Davies (The Chemical Society, London, 1975), Vol. 2, p. 151.

${ }^{19}$ G. Williams and D. C. Watts, Trans. Faraday Soc. 67, 197 (1971).

${ }^{20}$ G. Williams, Adv. Polym. Sci. 33, 60 (1979).

${ }^{21}$ A. Arbe, D. Richert, J. Colmenero, and B. Farago, Phys. Rev. E 54, 3853 (1996).

${ }^{22}$ E. Donth, K. Schröther, and S. Kahle, Phys. Rev. E 60, 1099 (1999).

${ }^{23}$ A. Arbe, D. Richert, J. Colmenero, and B. Farago, Phys. Rev. E 60, 1103 (1999).

${ }^{24}$ F. Garwe, A. Schönhals, H. Lockwenz, M. Beiner, K. Schröther, and E. Donth, Macromolecules 29, 247 (1996).

${ }^{25}$ G. Oster, A. Perelson, and A. Katchalsky, Nature 234, 393 (1971).

${ }^{26}$ J. W. Forrester, Principles of Systems (Wright, Cambridge, MA, 1968).

${ }^{27}$ D. C. Karnopp, D. L. Margolis, and R. C. Rosenberg, System Dynamic (Wiley, New York, 2006).

${ }^{28}$ G. Williams and D. C. Watts, Trans. Faraday Soc. 66, 80 (1970).

${ }^{29}$ R. Kohlrausch, Ann. Phys. 167, 179 (1854).

${ }^{30}$ D. W. Davidson and R. H. Cole, J. Chem. Phys. 18, 1417 (1950).

${ }^{31}$ D. W. Davidson and R. H. Cole, J. Chem. Phys. 19, 1464 (1951).

${ }^{32}$ S. Havriliak and S. Negami, J. Polym. Sci., Part C: Polym. Symp. 14, 99 (1966).

${ }^{33}$ S. Havriliak and S. Negami, Polymer 8, 161 (1967).

${ }^{34}$ T. Blochowicz, C. Tschirwitz, S. Benkhof, and E. A. Rössler, J. Chem. Phys. 118, 7544 (2003).

${ }^{35}$ R. Bergman, F. Alvarez, A. Alegria, and J. Colmenero, J. Chem. Phys. 109, 7546 (1998).

${ }^{36}$ T. Hecksher, N. B. Olsen, K. Niss, and J. C. Dyre (unpublished).

${ }^{37}$ K. Niss, C. Dalle-Ferrier, G. Tarjus, and C. Alba-Simionesco, J. Phys.: Condens. Matter 19, 076102 (2007).

${ }^{38}$ R. Bergman, AIP Conf. Proc. 1027, 1291 (2008);

${ }^{39}$ R. Bergman, J. App.. Phys. 88, 1356 (2000).

${ }^{40}$ T. Blochowicz and E. A. Rossler, Phys. Rev. Lett. 92, 225701 (2004).

${ }^{41}$ P. Debye, Z. Phys. 29, 401 (1928).

${ }^{42}$ K. S. Cole and R. S. Cole, J. Chem. Phys. 9, 341 (1941).

${ }^{43}$ In this paper we use "epsilon infinity" $\varepsilon_{\infty}$ in the meaning of being the high-frequency plateau value reached by dielectric spectroscopy (i.e., in the megahertz range). This means that it is not directly given by the refractive index, $\varepsilon_{\infty} \neq n^{2}$. See Ref. 51 for a discussion on the difference.

${ }^{44}$ A. K. Jonscher, Nature (London) 250, 191 (1974)

${ }^{45}$ A. K. Jonscher, Nature (London) 267, 673 (1977).

${ }^{46}$ A. K. Jonscher, Dielectric Relaxation in Solids (Chelsea, London, 1983).

${ }^{47}$ J. R. Macdonald, Impedance Spectroscopy (Wiley, New York, 1987).

${ }^{48}$ T. Christensen and N. B. Olsen, J. Non-Cryst. Solids 172, 357 (1994).

${ }^{49}$ A. Gemant, Trans. Faraday Soc. 31, 1582 (1935).

${ }^{50}$ E. A. DiMarzio and M. Bishop, J. Chem. Phys. 60, 3802 (1974).

${ }^{51}$ K. Niss, B. Jakobsen, and N. B. Olsen, J. Chem. Phys. 123, 234510 (2005).

${ }^{52}$ A. Kudlik, S. Benkhof, T. Blochowicz, C. Tschirwitz, and E. Rössler, J. Mol. Struct. 479, 201 (1999).

${ }^{53}$ P. Lunkenheimer, R. Wehn, T. Riegger, and A. Loidl, J. Non-Cryst. Solids 307-310, 336 (2002).

${ }^{54}$ P. K. Dixon, L. Wu, S. R. Nagel, B. D. Williams, and J. P. Carini, Phys. Rev. Lett. 65, 1108 (1990).

${ }^{55}$ A. Hofmann, F. Kremer, E. W. Fischer, and A. Schönhals, Disorder Effects on Relaxational Processes, edited by R. Richert and A. Blumen (Springer, Berlin, 1994), p. 308.

${ }^{56}$ U. Schneider, R. Brand, P. Lunkenheimer, and A. Loidl, Phys. Rev. Lett. 
84, 5560 (2000).

${ }^{57}$ P. Lunkenheimer and A. Loidl, Chem. Phys. 284, 205 (2002).

${ }^{58}$ K. L. Ngai, P. Lunkenheimer, C. Leon, U. Schneider, R. Brand, and A. Loidl, J. Chem. Phys. 115, 1405 (2001).

${ }^{59}$ P. Lunkenheimer, L. C. Pardo, M. Koehler, and A. Loidl, Phys. Rev. E 77, 031506 (2008).

${ }^{60}$ N. B. Olsen, J. Non-Cryst. Solids 235-237, 399 (1998).

${ }^{61}$ N. B. Olsen, T. Christensen, and J. C. Dyre, Phys. Rev. E 62, 4435 (2000).

${ }^{62}$ J. C. Dyre and N. B. Olsen, Phys. Rev. Lett. 91, 155703 (2003).

${ }^{63}$ A. Mandanici, W. Huang, M. Cutroni, and R. Richert, Philos. Mag. 88,
3961 (2008).

${ }^{64}$ G. Diezemann, U. Mohanty, and I. Oppenheim, Phys. Rev. E 59, 2067 (1999).

${ }^{65}$ B. Igarashi, T. Christensen, E. H. Larsen, N. B. Olsen, I. H. Pedersen, T. Rasmussen, and J. C. Dyre, Rev. Sci. Instrum. 79, 045106 (2008).

${ }^{66}$ B. Igarashi, T. Christensen, E. H. Larsen, N. B. Olsen, I. H. Pedersen, T. Rasmussen, and J. C. Dyre, Rev. Sci. Instrum. 79, 045105 (2008).

${ }^{67}$ R. Richert, Rev. Sci. Instrum. 67, 3217 (1996).

${ }^{68}$ C. Hansen and R. Richert, J. Phys.: Condens. Matter 9, 9661 (1997).

${ }^{69}$ W. Huang, S. Shahriari, and R. Richert, J. Chem. Phys. 123, 164504 (2005). 